\title{
Load balanced clustering protocol for enhancing the lifetime of wireless sensor network
}

\author{
Rajveer Kaur ${ }^{1 *}$ and Gurjinder Kaur ${ }^{2}$ \\ M Tech Student, Department of Computer Science and Engineering, Sant Longowal Institute of Engineering and \\ Technology, Longowal, Punjab, India ${ }^{1}$ \\ Associate Professor, Department of Computer Science and Engineering, Sant Longowal Institute of Engineering and \\ Technology, Longowal, Punjab, India ${ }^{2}$
}

\section{C2018 ACCENTS}

\begin{abstract}
In this paper, a grid-based load balanced clustering protocol $(\mathrm{LBCP})$ is proposed to prolong the network lifetime of wireless sensor network (WSN). The operations of LBCP include the division of the network into regular grids, maximum normalized residual energy-based cluster head $(\mathrm{CH})$ selection, and rotation of the role of $\mathrm{CH}$ for equal distribution of energy load among sensor nodes (SNs). In each grid, SNs send data to their respective CH in their allotted time slot. CHs then processes and transmits data to the base station (BS) using the code division multiple access (CDMA) technique. In LBCP, square grids are formed instead of circular clusters to avoid any voids between clusters. Numerous simulations are carried out to evaluate the performance of $\mathrm{LBCP}$ and the obtained results are compared with low energy adaptive clustering hierarchy (LEACH) protocol. The simulation results showed that LBCP can extend the network lifetime up to $45 \%, 48 \%$ and $83 \%$ more than $\mathrm{LEACH}$ in an area of $100 \mathrm{~m} \times 100 \mathrm{~m}, 200 \mathrm{~m} \times 200 \mathrm{~m}$ and $300 \mathrm{~m} \times 300 \mathrm{~m}$ respectively. In $\mathrm{LBCP}$, the energy of SNs is utilized in an efficient manner so that they remain alive for a longer time which results in the prolonged network lifetime. Hence, it can be concluded that the LBCP has better CH selection algorithm which helps in longer lifetime of WSN.
\end{abstract}

\section{Keywords}

LEACH, Load balanced clustering protocol, Energy efficiency, Network lifetime.

\section{Introduction}

Wireless sensor network (WSN) consisting the large number of sensor nodes (SNs) which are deployed throughout the network with at least one sink node or base station (BS). All SNs can perform data detecting, information handling and wireless communication functions. The BS is gathering data from these SNs [1, 2]. However, energy sources in SNs are limited and they are generally deployed in the remote area. So, changing the battery is sometime not possible due to harsh environment conditions like in volcano monitoring, forest monitoring etc. Therefore, the efficient utilization of energy resources in WSN is the main research issue. Along with the advances in micro electro mechanical system, routing protocols plays a vital role in the conservation of energy. Many such routing protocols have been proposed in the literature to utilize the limited energy in an efficient way. Routing protocols are divided into flat, hierarchical and location-based routing protocols based on the network structure.

*Author for correspondence
Hierarchical routing protocols are more energy efficient as compared to other routing protocols [3].

In the hierarchical protocol, SNs are grouped together and clusters are formed. In each cluster, one SN is selected as a cluster head $(\mathrm{CH})$ which collects data from member nodes (MNs). Then, $\mathrm{CH}$ aggregates and processes the data into information. At last, $\mathrm{CH}$ transmits the information to BS.

Low energy adaptive clustering hierarchy (LEACH) is the main hierarchical routing protocol which enhances the energy efficiency [4-7]. CHs are selected randomly in each cluster that means any $\mathrm{SN}$ with high or low energy can be chosen as a $\mathrm{CH}$. The role of $\mathrm{CH}$ is rotated and randomized for dispersion of energy needs among the SNs of the network. $\mathrm{CH}$ is suitable for homogeneous network and hierarchy is relatively simple [8]. Allocation of time division multiple access (TDMA) schedule by $\mathrm{CH}$ allows the SNs to go into sleep mode so that intracluster collisions will not occur and increases battery lifetime of SNs. Implementation of $\mathrm{LEACH}$ is 
impossible in large networks due to the chance of no $\mathrm{CH}$ in the closed area [9]. Limitations of LEACH protocol area as (1) LEACH expect that SNs dependably have data to send and there is a relationship between data of SNs that are found near each other. CHs are not consistently circulated within the cluster that implies $\mathrm{CHs}$ can be situated at the edges of the cluster, and a few SNs might not have any $\mathrm{CHs}$ in their nearby environment. (2) In LEACH protocol, sometimes imbalanced clusters are formed in which energy consumption is not balanced due to a random selection of $\mathrm{CH}[6,10]$.

In the literature, numerous researchers have proposed their work to enhance the energy efficiency and network lifetime. Ahmed et al. [1] have presented the wireless sensor characteristics and architecture to design functional WSN and get the highest throughput by using minimum low-cost resources. In $[3,10]$ a thorough survey of routing protocols and strategies in view of the network structure and data transmission utilized as a part of the wireless sensor network (WSN) has been presented for development in the execution of WSN. Kalantari and Ekbatanifard [11] proposed a new method in which nearest SN was selected as a $\mathrm{CH}$ in clusters that reduced the energy consumption and prevented the early death of nodes. Nguyen et al. [12] have proposed two approaches distance-based LEACH and distance-based energy aware $\mathrm{LEACH}$ for $\mathrm{CH}$ selection in view of separation between candidate nodes to BS and furthermore considered the remaining energy of $\mathrm{SN}$ higher than the average remaining energy of SNs in the system. Moussa et al. [13] had minimized the distance between SNs and divided the network into clusters having ordinary nodes and intense nodes $\mathrm{CHs}$. Then, located the CHs into a normal of the area of ordinary nodes which saves more energy. Consequently, various authors have proposed different algorithms for $\mathrm{CH}$ selection to reduce energy consumption that can enhance the network lifetime [14-24]. Aforementioned literature can significantly enhance the network lifetime and efficiency, but investigations on load balance clustering is still pending.

In this paper, a grid-based load balanced clustering protocol (LBCP) is proposed for proper $\mathrm{CH}$ selection to the enhancement of network lifetime. Grids are created in such a manner in which a load of energy is balanced among the SNs of the network. SN with maximum normalized energy is selected as a $\mathrm{CH}$ within the grid. Further, LBCP is compared with the LEACH protocol that shows WSN has longer network lifetime by using LBCP as compared to network lifetime by using LEACH. Main objective of this paper is to design an efficient $\mathrm{CH}$ selection algorithm for extending the network lifetime by balancing the load of SNs in WSN [25-29].

Rest of the paper is organized as follows: section 2 describes the network model and energy model used in LBCP. Section 3 presenting the LBCP with its architecture, phases and $\mathrm{CH}$ selection algorithm. Section 4 shows the result analysis and finally, conclusions have been presented in section 5 .

\section{Proposed load balanced clustering protocol}

2.1Network model

Assumes that a WSN with an area of $a \times a$ square units, where $a$ denotes the length of the network. $N$ SNs and a sink node (i.e. BS) are deployed randomly in the sensor network. Sink node has unlimited storage, computation and battery power. We accept that the sink node is situated at the focal point of the system. SNs are deployed randomly in the plane area. All SNs are homogeneous i.e. capabilities of computation, communication and initial energy are same and predefined.

\subsection{Energy model}

In this work, a simple radio model is used which is given by Heinzelman et.al $[5,6]$ for evaluating energy dissipation. Assumes that radio consumes energy $E_{\text {elec }}$ to run the circuits of transmitter or receiver and $\epsilon_{a m p}$ is the energy dissipated for transmision amplifier. In the model, both the free space and the multipath blurring channel models are utilized for experimentation, contingent upon the separation between the transmitter and the receiver. If the separation is not as much as a threshold, the free space $(\boldsymbol{f} s)$ model is used; otherwise, the multipath (mp) model is utilized. To communicate something specific of $k$ bits to a separation $d$ utilizing radio model, radio uses:

$E_{T x}(k, d)=\left\{\begin{array}{c}E_{\text {elect }} * k+\epsilon_{f s} * k * d^{2}, \text { ifd }<d_{0} \\ E_{\text {elect }} * k+\epsilon_{m p} * k * d^{4}, \text { if } d \geq d_{0}\end{array}(1)\right.$

Where threshold $d_{0}=\sqrt{\epsilon_{f s} / \epsilon_{m p}}$

To get a message, the radio uses:

$E_{R x}(k)=E_{\text {elec }} * k$

Essential factors like digital coding, separating and flag spreading on which electronics energy, $E_{\text {elect }}$ are reliant and amplifier energy, $\epsilon_{f s} d^{2}$ or $\epsilon_{m p} d^{4}$ relies upon separation to the receiver and bit mistake rate which is adequate. 


\section{Description of load balanced clustering protocol with its operation}

LBCP solves the problems of LEACH and energy consumption is balanced among the SNs by (i) partitioning the WSN into square-shaped grids that cover the whole WSN; (ii) selecting the optimum $\mathrm{CH}$ in each grid based on the maximum normalized residual energy; (iii) rotating the role of $\mathrm{CH}$ after one round so that energy load will be equally distributed between SNs. Thus, only one SN should not be overloaded which leads to survive more time or its energy will not be consumed quickly. Because LEACH network lifetime is short, WSN becomes dead early then, all SNs of the network have large residual energy. Reduction in the energy of one SN causes the network connectivity to be broken. To overcome this, LBCP extends the network lifetime in the way in which the maximum energy of every SN before the network dies will be consumed. Therefore, residual energy reduces more as compared to LEACH. It implies when the network dies it will have the very little amount of energy as the energy of the SNs are utilized in efficient way in LBCP.

Diagram of LBCP is shown in Figure 1. The diagram shows the division of the network into 12 grids, deployment of $\mathrm{SNs}$ in each grid, gathering sensed data from MNs by $\mathrm{CH}$ within the grid. $\mathrm{CHs}$ processes and aggregates data to central BS or sink.

The LBCP's operation is partitioned into rounds and in each round, there are three phases: information collection phase, $\mathrm{CH}$ selection phase and data collection phase as shown in Figure 2. In the first phase, BS collects information from all SNs and creates grids based on received information. In second phase, BS selects $\mathrm{CH}$ in each grid and $\mathrm{CH}$ makes a TDMA schedule to SNs. Data transferring from the SNs to $\mathrm{CHs}$ and continuously from $\mathrm{CH}$ to BS in the third phase. Details of each phase of LBCP algorithm are described in next section.

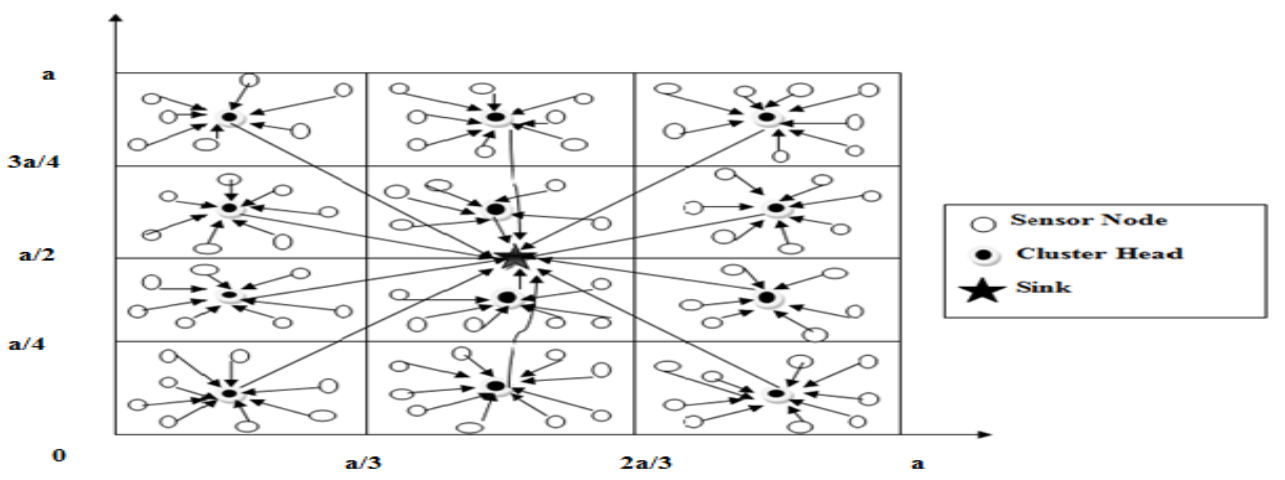

Figure 1 LBCP architecture

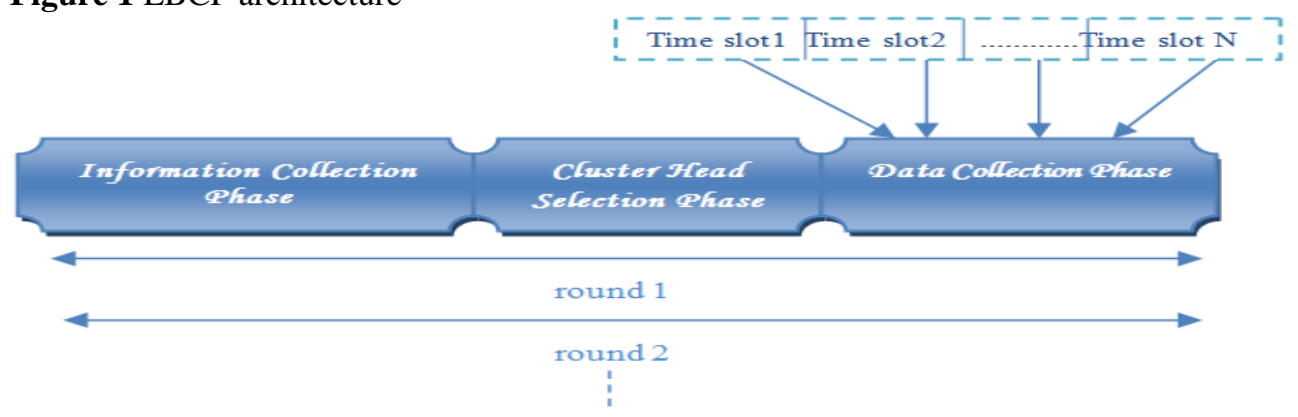

Figure 2 Operation of LBCP

\subsection{Information collection phase}

Information collection phase occurs one time before the first round. In this phase, information of all $\mathrm{SNs}_{\mathrm{N}}$ is collected by BS and network is partitioned into 12 grids. Firstly, BS gives the information of its ID and location to all SNs by sending a request message i.e. req_msg. All SNs receives the req_msg and send self_info_msg that gives the information of their ID, location and residual energy to BS. Then, BS receives the self_info_msg from all SNs. All SNs are set as an ordinary node $(\mathrm{ON})$ on the basis of received information. After that, the network is partitioned into 12 equal and square shaped grids on the basis of received information. 
3.2Cluster head selection phase

In $\mathrm{CH}$ Selection phase, one optimal $\mathrm{CH}$ is selected from each grid. At first, BS calculates the residual energy of all SNs of each grid. BS constructs the arrays of grids $G_{i}$ contains the ID of SNs that belongs to the grid $i$. In grid wise manner, BS takes the minimum and maximum value of residual energy of SNs in a grid. Then it normalizes the SN's energy in the range of 0 and 1 . Normalization is done according to two conditions. If the standard deviation of SN's residual energy is zero, then its normalized value of energy is taken as 1 otherwise energy is normalized according to Equation 3.

$N E=((R E-\min )) /((\max -\min ))$

Where $N E$ denotes the normalized energy, $R E$ denotes the residual energy of $\mathrm{SN}, \min$ denotes minimum energy of SNs in the grid, max denotes the maximum energy of SNs in grid. BS selects the SN with maximum normalized energy as a $\mathrm{CH}$ in a grid. BS send CH_info_msg contains ID of $\mathrm{CHs}$ to all $\mathrm{CHs}$ in the network. CHs receives the message from BS. Then, if the $\mathrm{SN}$ is a $\mathrm{CH}$, then $\mathrm{CH}$ broadcasts the advertisement message (Adv_msg) contains its ID to all the SNs. After receiving Adv_msg, SNs choose their $\mathrm{CHs}$ and they send the join_req_msg to their chosen $\mathrm{CH}$. Then, $\mathrm{CH}$ accepts the join_req_msg from SNs and now it knows about the MNs of its grid. At last of this phase, TDMA schedule for each MN is created by $\mathrm{CH}$.

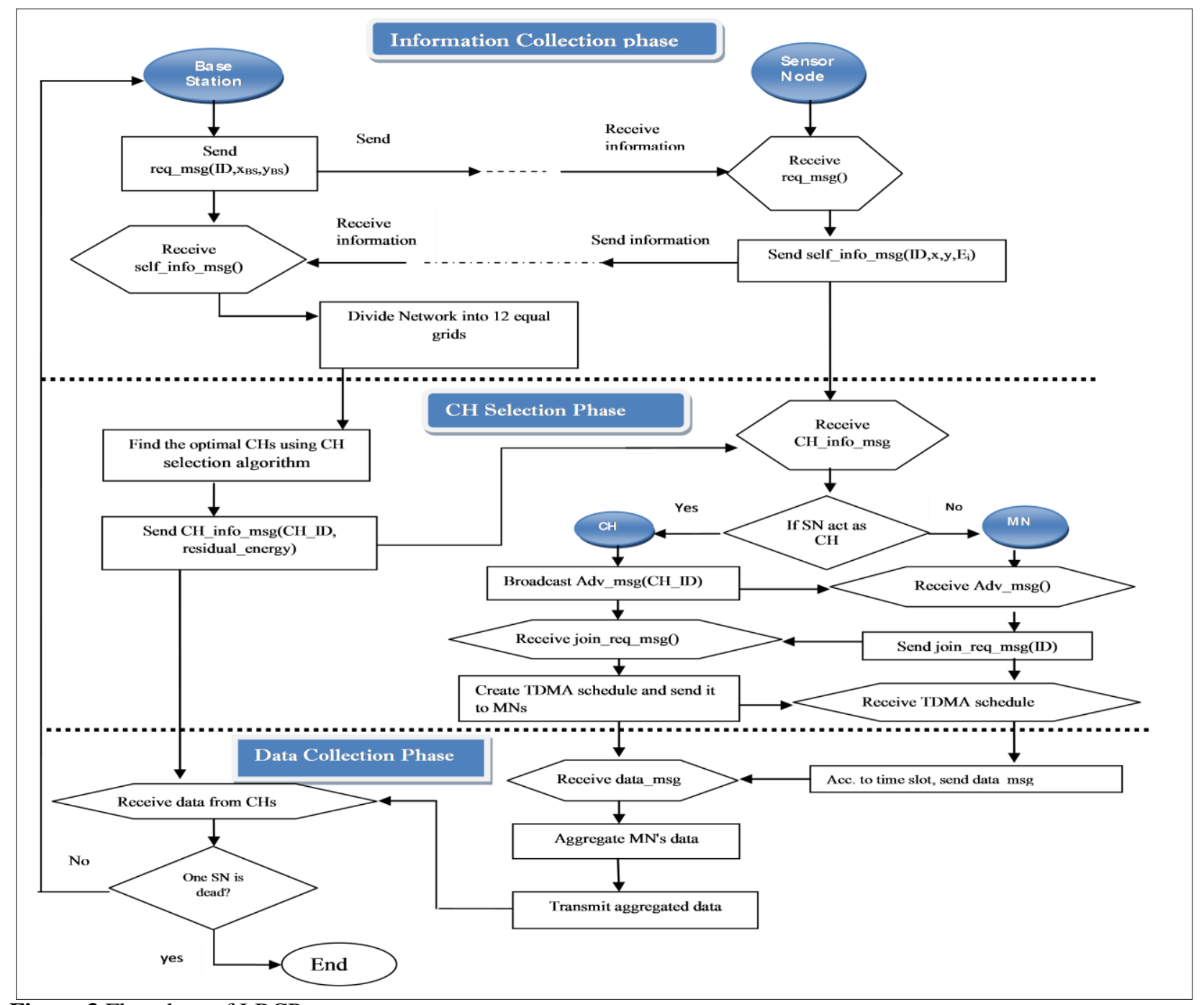

Figure 3 Flowchart of LBCP 


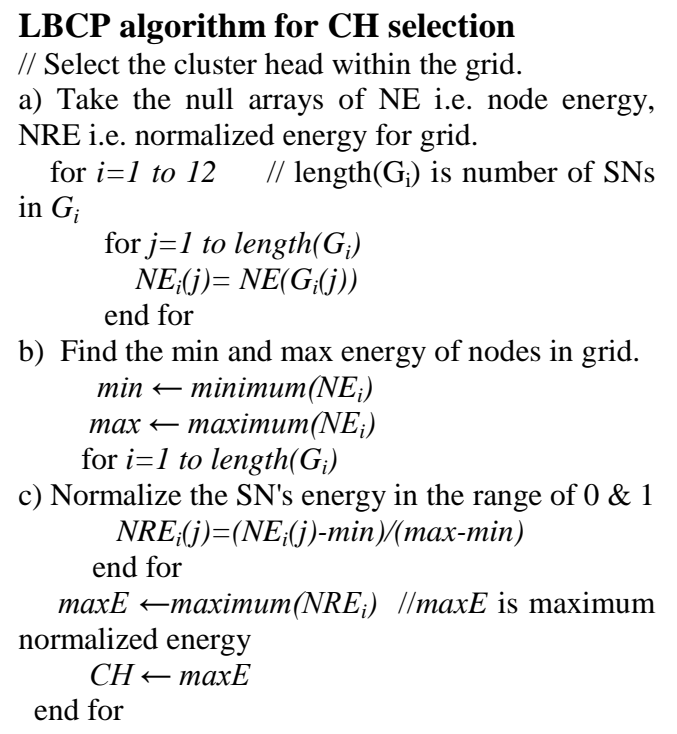

3.3Data collection phase

As there is a correlation in between data of MNs, an aggregation is performed by $\mathrm{CHs}$ to reduce this correlation. In this phase, MNs send the detected information to $\mathrm{CHs}$ during their time slot according to the TDMA schedule allocated by $\mathrm{CHs}$. $\mathrm{MN}$ is active only during its time slot. To receive data from all MNs within the grid, the receiver of each $\mathrm{CH}$ should be on. Different CDMA are used by grids for communication so that interference of $\mathrm{CHs}$ belonging to other grids can be reduced. $\mathrm{CHs}$ accept the data from MNs. Then, CHs processes and transmits the aggregated data to the $\mathrm{BS}$. BS receives the data from CHs. If one of the SNs become dead, then the whole network ends otherwise next round starts and process from $\mathrm{CH}$ selection phase and data collection phase begin again. Flowchart of LBCP operation is shown in Figure 3.

\section{Results and discussion}

\subsection{Performance evaluation}

MATLAB software is used as a simulation platform to implement the LEACH protocol and LBCP (proposed protocol). Performance of the LBCP is measured from three aspects: the total energy consumption, the residual energy and lifetime of the network.

Network Lifetime: Network lifetime shows round $r$ in which first $\mathrm{SN}$ of the network dies. Some researchers considered the same for evaluating network lifetime [11],[30-33].

Energy Consumption: It shows the total energy consumed by WSN.

TotalEN $(r-1)=T E_{\text {ofnodes }}-\sum_{i=1}^{100} N E(i)$
Where TotalEN $(r-1)$ denotes the total energy consumption of the whole network up to $(r-1)$ round, $T E_{\text {ofnodes }}$ denotes the sum of initial energy of all nodes and $N E$ denotes the energy of SN.

Energy utilization: It shows the amount of energy utilized by WSN.

$R E_{\text {ofnet }}=T E_{\text {ofnodes }}-\operatorname{TotalEN}(r-1)$

Where $R E_{\text {ofnet }}$ denotes the residual energy of the whole network.

Table 1 shows the parameters used for simulating results. Three different areas like $100 \mathrm{~m} \times 100 \mathrm{~m}$, $200 \mathrm{~m} \times 200 \mathrm{~m}$ and $300 \mathrm{~m} \times 300 \mathrm{~m}$ are taken for network.

Table 1 Simulation parameters

\begin{tabular}{lll}
\hline S. No. & Parameter & Value \\
\hline 1 & No_of_nodes, N & 100 \\
2 & No_of_rounds & 10000 \\
3 & Initial_energy & $0.5 \mathrm{~J}$ \\
4 & Transmit_energy, $\mathrm{E}_{\text {elect }}$ & $5 \mathrm{~nJ} / \mathrm{bit}$ \\
5 & Receive_energy, $\mathrm{E}_{\text {elect }}$ & $5 \mathrm{~nJ} / \mathrm{bit}$ \\
6 & Freespace_energy, $\epsilon_{\mathrm{fs}}$ & $0.0013 \mathrm{pJ} / \mathrm{bit} / \mathrm{m}^{2}$ \\
7 & Multipath_energy, $\epsilon_{\mathrm{mp}}$ & $0.0013 \mathrm{pJ} / \mathrm{bit}^{4} / \mathrm{m}^{4}$ \\
8 & Aggregate_energy & $0.5 \mathrm{~nJ} / \mathrm{bit} / \mathrm{signal}$ \\
9 & Packet_length $(\mathrm{CH}$ to BS) & $6400 \mathrm{bits}$ \\
10 & Ctrpacket_length, $(\mathrm{SN}$ to $\mathrm{CH})$ & $200 \mathrm{bits}$ \\
\hline
\end{tabular}

4.2 Average network lifetime

We use multiple scenarios for different network sizes and in each scenario; there is a change in location of SNs. In each scenario, values of network lifetime using LBCP are much higher than values of network lifetime using LEACH. Then, the average (Avg) network lifetime is calculated.

As appears in Figure 4, in the network size of $100 \mathrm{~m} \times 100 \mathrm{~m}$ by using LBCP, on average first node dies in the $7752^{\text {nd }}$ round and by using $\mathrm{LEACH}$ protocol, on average first node dies in the $5582^{\text {nd }}$ round. Average network lifetime in LBCP is $45 \%$ more than average network lifetime in LEACH. Similarly, in the field of $200 \mathrm{~m} \times 200 \mathrm{~m}$, on average first node dies in the $7733^{\text {rd }}$ round in LBCP and on average first node dies in the $5195^{\text {th }}$ round in LEACH protocol. Average network lifetime in LBCP is $48 \%$ more than average network lifetime in LEACH. Similarly, in the field of $300 \mathrm{~m} \times 300 \mathrm{~m}$, on average first node dies in the $7722^{\text {nd }}$ round by LBCP and on average first node dies in the $4215^{\text {th }}$ round by LEACH protocol. Average network lifetime in LBCP is $83 \%$ more than the average network lifetime in LEACH. There is a small drop in network lifetime of WSN in different areas by using LBCP. In our proposed LBCP and $\mathrm{LEACH}$, as an area of network 
increases, the lifetime of the network decreases. Because when the area is small, then the distance between SNs is also very small and each SN consumes a small amount of energy. Therefore, it does not die quickly and has a long lifetime. Figure 4 shows a simulation result of LBCP and LEACH that compares the average network lifetime versus area. This shows that network lifetime using LBCP is more than network lifetime in LEACH. In our method, clustering is done by partitioning the network into equal grids and these grids are balanced in which $\mathrm{CHs}$ are selected from each grid. $\mathrm{CH}$ 's role is rotated after one round so that energy load is distributed equally to all SNs and thus, each SN has a long lifetime. In LEACH protocol, balanced clusters are not shaped, and some $\mathrm{CHs}$ are chosen in one region of the network. When low energy $\mathrm{SN}$ is selected as $\mathrm{CH}$, it dies early, and network lifetime is small.

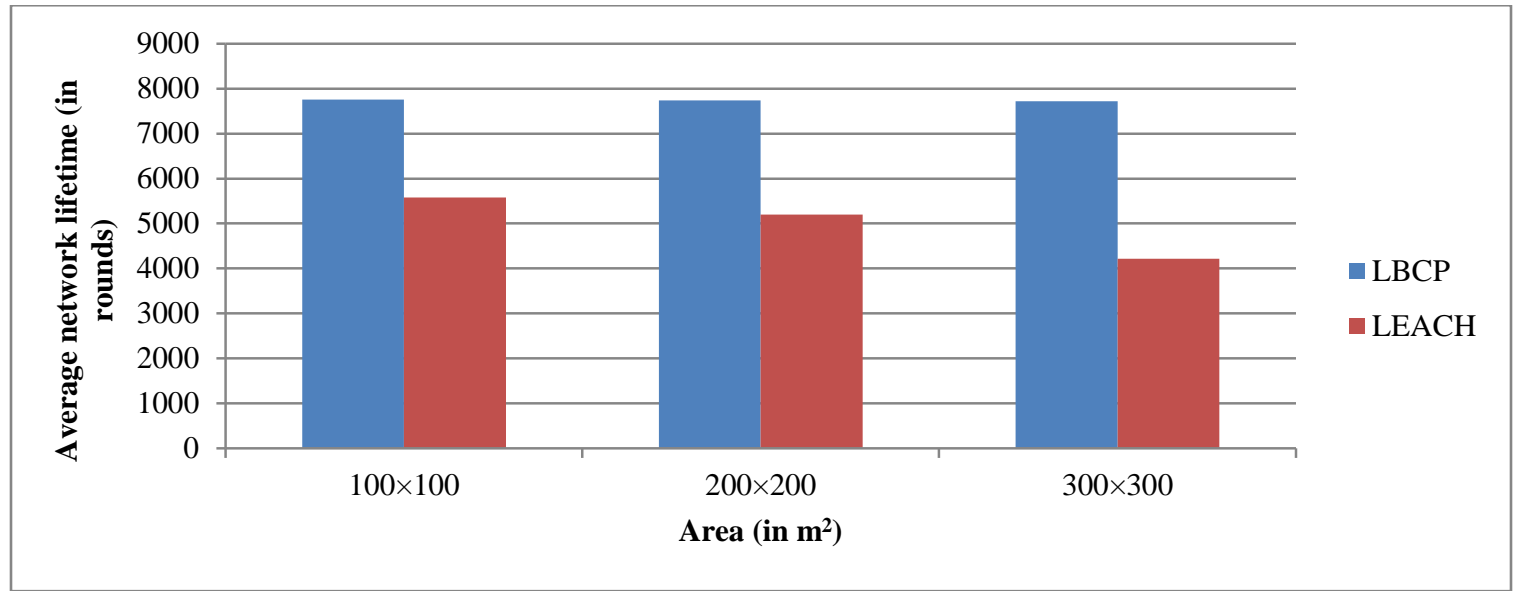

Figure 4 Average network lifetime of WSN in different areas

\subsection{Average residual energy}

Residual energy of the network is calculated after the first node dies (FND). For different network sizes, by using LBCP results of multiple scenarios gives the values of residual energy of network which are higher than values of residual energy of the network by using LEACH. As shown in Figure 5, in the field of $100 \mathrm{~m} \times 100 \mathrm{~m}, 200 \mathrm{~m} \times 200 \mathrm{~m}$ and $300 \mathrm{~m} \times 300 \mathrm{~m}$, the average residual energy of network in LBCP are $1.1866 \mathrm{~J}, 1.2976 \mathrm{~J}$ and $1.3488 \mathrm{~J}$ respectively.
The average residual energy of network in LEACH are $14.026 \mathrm{~J}, 16.50 \mathrm{~J}$ and $22.80 \mathrm{~J}$ in the field of $100 \mathrm{~m} \times 100 \mathrm{~m}, \quad 200 \mathrm{~m} \times 200 \mathrm{~m}, \quad 300 \mathrm{~m} \times 300 \mathrm{~m}$ respectively. It is observed that the average residual energy of WSN using LBCP is less than that of WSN using $\mathrm{LEACH}$ in the respective field. With increase in area, network lifetime and residual energy decreases.

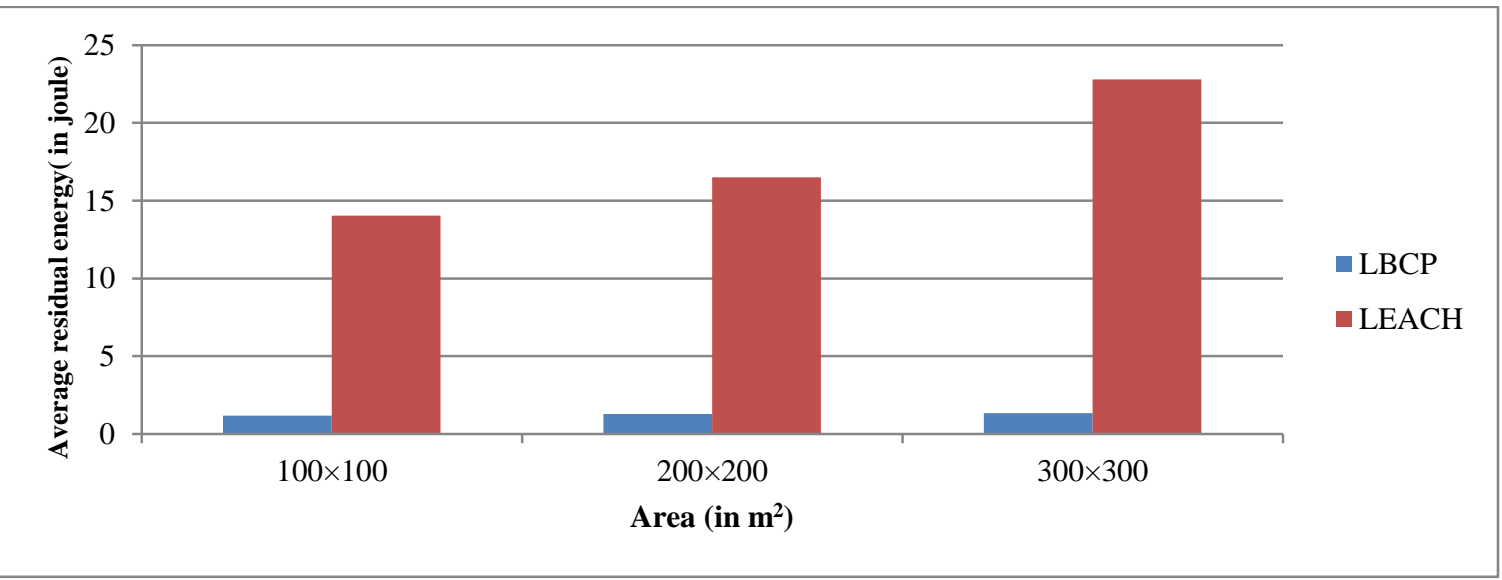

Figure 5 Average residual energy of network in different areas 
Kaur and Kaur.

If the network lifetime is more, then it means network works for a large number of rounds. In LBCP, when the network lifetime is over, the residual energy of the whole network becomes very low and the maximum energy of every $\mathrm{SN}$ is consumed. This is because LBCP utilizes the energy of SNs in an efficient way. In LEACH protocol, network lifetime is very small. When the network dies, each $\mathrm{SN}$ has a large amount of remaining energy and there is no maximum energy consumption of the network.

\subsection{Average energy consumption}

Energy consumed by WSN in each scenario is smaller in LBCP as compared to LEACH. Average energy consumption of network of these scenarios in
LBCP is also smaller than LEACH. Figure 6 to Figure 8 shows the amount of average energy consumption of WSN having network size $100 \mathrm{~m} \times 100 \mathrm{~m}, \quad 200 \mathrm{~m} \times 200 \mathrm{~m}$ and $300 \mathrm{~m} \times 300 \mathrm{~m}$ respectively.

In our proposed LBCP, optimal $\mathrm{CHs}$ are selected from each grid based on maximum normalized residual energy which ensures that $\mathrm{CH}$ has enough energy to perform its all operations. Regular grids are created for equal energy distribution to all SNs. Due to balanced energy consumption; a small amount of energy of SNs is consumed. But in LEACH protocol, imbalanced clusters are formed and energy consumption of SNs is imbalanced because $\mathrm{CHs}$ are selected randomly.

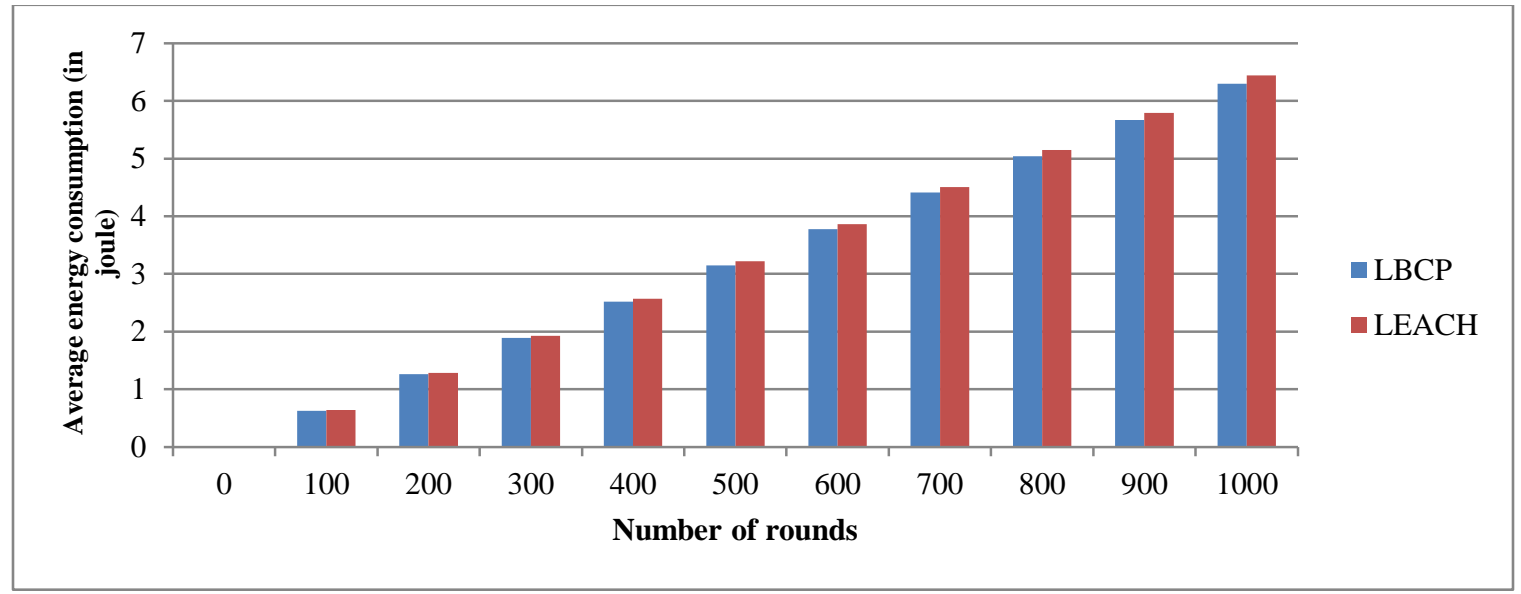

Figure 6 Average energy consumption of WSN in network size $100 \mathrm{~m} \times 100 \mathrm{~m}$

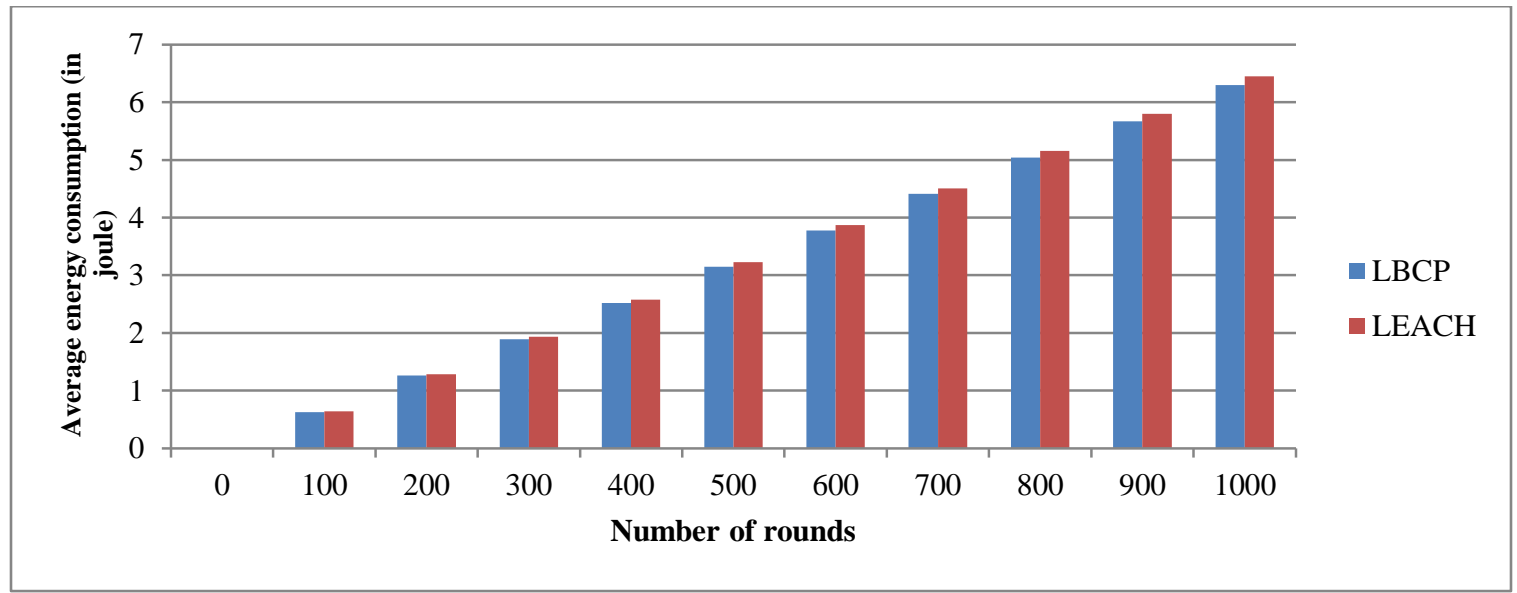

Figure 7 Average energy consumption of WSN in network size $200 \mathrm{~m} \times 200 \mathrm{~m}$ 


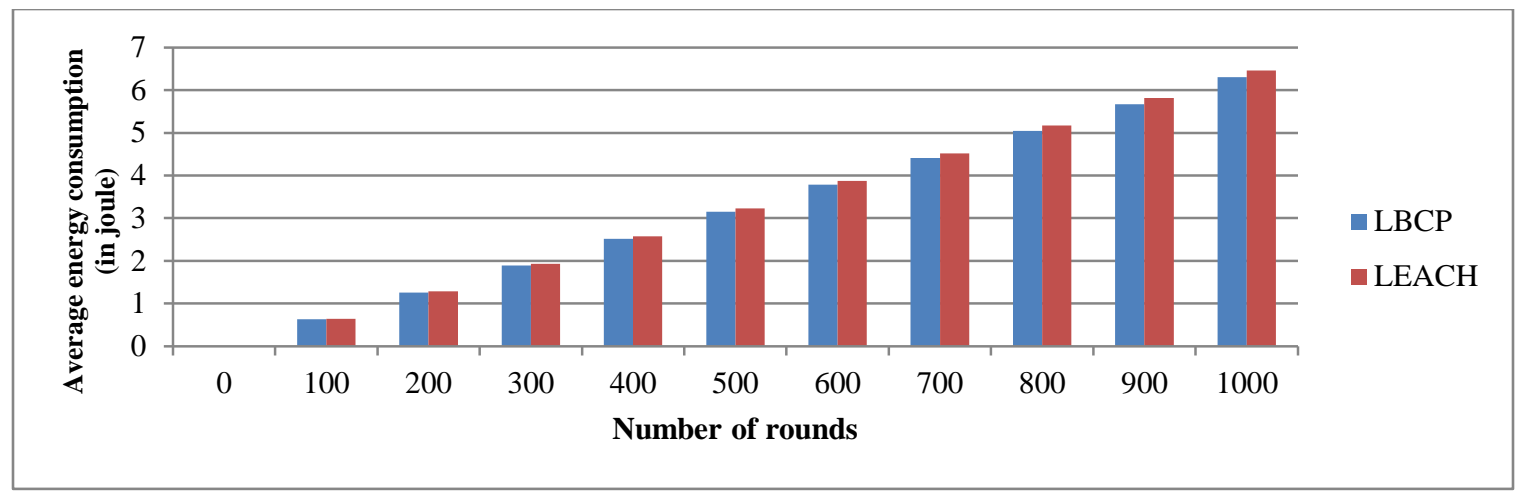

Figure 8 Average energy consumption of WSN in network size $300 \mathrm{~m} \times 300 \mathrm{~m}$

\section{Conclusion}

An important technique clustering can take an interest in entire network lifetime and energy efficiency in WSN. The proposed protocol LBCP aims to utilize the energy efficiently and enhance the network lifetime. LBCP provide solutions to the main problems of LEACH. The strategy of $\mathrm{CH}$ selection in clusters of WSN enhances the performance of LEACH protocol. LBCP divides the WSN into square grids and selects $\mathrm{CH}$ from each grid that makes the load balancing among SNs. LBCP algorithm select the optimal $\mathrm{CH}$ based on maximum normalized energy within the grid. Part of $\mathrm{CH}$ is turned after one round for equal distribution of energy load between SNs so that SNs lives for a long time and their network lifetime increases. Energy consumption of WSN is better in LBCP as compared to LEACH. Network lifetime is longer in LBCP as compared to LEACH. Using LBCP, WSN dies most probably after 7000 rounds but using LEACH it dies after 5000 rounds. In LBCP, we utilize the energy of SNs efficiently and consume maximum energy before the network dies. But in LEACH, we cannot use the maximum energy of SNs before network dies because it cannot be used efficiently and network lives for a short time. Energy efficiency is higher in LBCP as compared to LEACH. The work presented in this paper has significant role in improvement of WSN. This work also has substantial scope for further research for improving the performance of other routing protocols. The future works includes the establishment of cluster and selection of $\mathrm{CHs}$ in cluster by using the proposed methodology by including some additional work.

\section{Acknowledgment}

None.

\section{Conflicts of interest}

The authors have no conflicts of interest to declare.

\section{References}

[1] Ahmed MR, Huang X, Sharma D, Cui H. Wireless sensor network: characteristics and architectures. World Academy of Science, Engineering and Technology, International Journal of Electrical, Computer, Energetic, Electronic and Communication Engineering. 2012; 6(12):1398-401.

[2] Agarwal D, Gupta A. Energy efficient clustering based 3-rank heterogeneous network model for wireless sensor network. In international conference on advances in computer engineering and applications 2015 (pp. 183-8). IEEE.

[3] Singh H, Singh D. Taxonomy of routing protocols in wireless sensor networks: a survey. In international conference on contemporary computing and informatics 2016 (pp. 822-30). IEEE.

[4] Reddy GR, Balaji S. A review on different types of LEACH protocol for wireless sensor networks. International Journal of Scientific Research in Computer Science, Engineering and Information Technology. 2017; 2(4):840-4.

[5] Heinzelman WR, Chandrakasan A, Balakrishnan H. Energy-efficient communication protocol for wireless microsensor networks. In annual Hawaii international conference on system sciences 2000 (pp. 1-10). IEEE.

[6] Heinzelman WB, Chandrakasan AP, Balakrishnan H. An application-specific protocol architecture for wireless microsensor networks. IEEE Transactions on Wireless Communications. 2002; 1(4):660-70.

[7] Arora VK, Sharma V, Sachdeva M. A survey on LEACH and other's routing protocols in wireless sensor network. Optik-International Journal for Light and Electron Optics. 2016; 127(16):6590-600.

[8] Upadhyay A, Kumar R, Tiwari SK. Modified LEACH protocol for sensor network. In international conference on futuristic trends on computational analysis and knowledge management 2015 (pp. 3014). IEEE.

[9] Liu X. A survey on clustering routing protocols in wireless sensor networks. Sensors. 2012; 12(8):1111353.

[10] Al-Karaki JN, Kamal AE. Routing techniques in wireless sensor networks: a survey. IEEE Wireless Communications. 2004; 11(6):6-28. 
[11] Kalantari M, Ekbatanifard G. An energy aware dynamic cluster head selection mechanism for wireless sensor networks. In international systems conference 2017 (pp. 1-8). IEEE.

[12] Nguyen TG, So-In C, Nguyen NG. Two energyefficient cluster head selection techniques based on distance for wireless sensor networks. In international computer science and engineering conference 2014 (pp. 33-8). IEEE.

[13] Moussa S, Darazi R, Atechian T, Demerjian J. Synchronized region based clustering for energy saving in wireless sensor networks. In international multidisciplinary conference on engineering technology 2016 (pp. 15-20). IEEE.

[14] Wang X, Zhang X, Chen G, Tian S. An adaptive and distributed clustering scheme for wireless sensor networks. In international conference on convergence information technology 2007 (pp. 522-7). IEEE.

[15] Ye M, Li C, Chen G, Wu J. EECS: an energy efficient clustering scheme in wireless sensor networks. In international conference on performance, computing, and communications 2005 (pp. 535-40). IEEE.

[16] Beiranvand Z, Patooghy A, Fazeli M. I-LEACH: an efficient routing algorithm to improve performance \& to reduce energy consumption in wireless sensor networks. In conference on information and knowledge technology 2013 (pp. 13-8). IEEE.

[17] Mohamed-Lamine M. New clustering scheme for wireless sensor networks. In international workshop on systems, signal processing and their applications 2013 (pp. 487-91). IEEE.

[18] Prabha D, Arora VK. A survey on LEACH and its descendant protocols in wireless sensor network. In international conference on communication, computing \& systems 2014 (pp. 162-7).

[19] Wang S, Yu J, Atiquzzaman M, Chen H, Ni L. CRPD: a novel clustering routing protocol for dynamic wireless sensor networks. Personal and Ubiquitous Computing. 2018; 22(3):545-59.

[20] Sabor N, Abo-Zahhad M, Sasaki S, Ahmed SM. An unequal multi-hop balanced immune clustering protocol for wireless sensor networks. Applied Soft Computing. 2016; 43:372-89.

[21] Liu X. Atypical hierarchical routing protocols for wireless sensor networks: a review. IEEE Sensors Journal. 2015; 15(10):5372-83.

[22] Shankar M, Sridar M, Rajani M. Performance evaluation of LEACH protocol in wireless network. International Journal of Scientific \& Engineering Research. 2012; 3(1):1-7.

[23] Gambhir S, Parul. OE-LEACH: an optimized energy efficient LEACH algorithm for WSNs. In international conference on contemporary computing 2016 (pp. 16). IEEE.

[24] Ray A, De D. Energy efficient cluster head selection in wireless sensor network. In international conference on recent advances in information technology 2012 (pp. 306-11). IEEE.
[25] Mahapatra RP, Yadav RK. Descendant of LEACH based routing protocols in wireless sensor networks. Procedia Computer Science. 2015; 57:1005-14.

[26] Singh SK, Kumar P, Singh JP. A survey on successors of LEACH protocol. IEEE Access. 2017; 5:4298-328.

[27] Al-Baz A, El-Sayed A. A new algorithm for cluster head selection in LEACH protocol for wireless sensor networks. International Journal of Communication Systems. 2018; 31(1):1-13.

[28] Sobti R. A comparative study on network structure based routing protocol and its variants in wireless sensor networks: a survey. International Journal of Computer Applications. 2015; 117(12):27-33.

[29] Braman A, Umapathi GR. A comparative study on advances in LEACH routing protocol for wireless sensor networks: a survey. International Journal of Advanced Research in Computer and Communication Engineering. 2014; 3(2):5683-90.

[30] Fu C, Jiang Z, Wei WE, Wei A. An energy balanced algorithm of LEACH protocol in WSN. International Journal of Computer Science Issues. 2013; 10(1):3549.

[31] Bai FE, Tian EZ. Energy-efficient clustering hierarchy model for wireless sensor network. In international conference on information science and engineering 2009 (pp. 2622-4). IEEE.

[32] Younis O, Krunz M, Ramasubramanian S. Node clustering in wireless sensor networks: recent developments and deployment challenges. IEEE Network. 2006; 20(3):20-5.

[33] Kaur G, Jain VK, Chaba Y. Detection and prevention of blackhole attacks in wireless sensor networks. In international conference on intelligent, secure, and dependable systems in distributed and cloud environments 2017 (pp. 118-26). Springer, Cham.

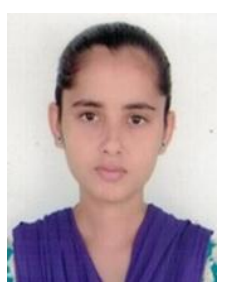

Miss. Rajveer Kaur is currently pursuing her M.Tech in department of Computer Science and Engineering (CSE) from Sant Longowal Institute of Engineering and Technology (SLIET) University, Longowal, Sangrur, Punjab, India. She has completed her B.Tech and Diploma in CSE from SLIET University. She has carried out work on Wireless Sensor Network.

Email: rajveerdhania25@gmail.com

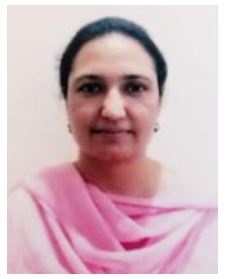

Mrs. Gurjinder Kaur is presently working as Associate Professor in department of Computer Science and Engineering at SLIET. She completed her B.E from Kyrgyzstan Technical University, Bishkek and M.S from BITS Pilani. She is pursuing Ph.D in area of Adhoc Networks. She has almost 20 years of experience in teaching. She has almost 20 national and international publications to her credit in reputed journals and conferences. 